\title{
Advanced Kaposi Sarcoma
}

National Cancer Institute

\section{Source}

National Cancer Institute. Advanced Kaposi Sarcoma. NCI Thesaurus. Code C156476.

Kaposi sarcoma that has spread extensively to other anatomic sites or is no longer responding to treatment. 